\title{
Osteoblast Cell Response to Oxide Films Formed on Nanograin 316L Stainless Steel Obtained by Two- dimensional Linear Plane-strain Machining
}

\author{
Mahdis Shayan $^{\mathrm{a}}$, Marzyeh Moradi ${ }^{\mathrm{b}}$, Anton Y. Plakseychuk ${ }^{\mathrm{c}}$, Ravi Shankar ${ }^{\mathrm{a}}$, Youngjae Chun $^{\mathrm{a}, \mathrm{d}}$ \\ ${ }^{a}$ Department of Industrial Engineering, University of Pittsburgh, 522 Benedum Hall, Pittsburgh, PA 15261, \\ USA \\ ${ }^{\mathrm{b}}$ Department of Mechanical Engineering and Materials Science, University of Pittsburgh, 522 Benedum Hall, \\ Pittsburgh, PA 15261, USA \\ ${ }^{\mathrm{c}}$ Bone and Joint Center at Magee-Womens Hospital of UPMC, 300 Halket St. Suite 1601-B, Pittsburgh, PA \\ 15213, USA \\ ${ }^{\mathrm{d}}$ Department of Bioengineering, University of Pittsburgh, 522 Benedum Hall, Pittsburgh, PA 15261, USA*
}

\begin{abstract}
The long-term success of orthopedic implants is strongly determined by their surface characteristics and the interaction of this surface with the surrounding biological environment. The biocompatibility of metallic biomaterials is mainly due to their ability to form an adherent oxide layer on their surface that is in direct contact with the biological environment. In this study, oxide layers were grown on ultra-fine grained 316L stainless steel samples, naturally, chemically and thermally. Samples in three different nanoscale average grain sizes were obtained by severe plastic deformation using linear plane-strain machining technique. Refining the grain size along with growing the oxide layer create surfaces with a wide variety of surface topography, roughness and chemistry. After chemical treatment, a chromium-enriched oxide with island-like topography was formed on the surface and substrates with smaller average grain size demonstrated rougher surfaces. After thermal treatment, micro-scaled oxide grains were formed on the surface and the amount of manganese oxide in its composition was increased. MC3T3 cell adhesion was greatly improved on the native oxide formed on the sample with the smallest average grain size. The oxide layer that is naturally formed on the surface demonstrated higher biocompatibility compared to both thermally and chemically formed oxides.
\end{abstract}

\footnotetext{
${ }^{*}$ Corresponding author. Tel.:+1 412-624-1193. Fax: +1 412-624-9831. E-mail address: yjchun@ pitt.edu
} 
Keywords: Nanograin, Osteoblast, Stainless steel oxide, Linear plane-strain machining (LSM), Severe plastic deformation (SPD)

\section{Introduction}

Increasing the average life expectancy and demands for orthopedic devices result in an urgent requirement to developing novel orthopedic devices with higher durability and functionality[1]. Metals present a wide range of favorable mechanical properties for load-bearing orthopedic applications compared to ceramic and polymer compositions; however, they are all inert and do not interact with the biological environment to bond with the bone and enhance the biological interface. Therefore, improving the biological function of metallic surface can open the window to design and develop novel durable orthopedic biomaterials[2, 3]. Biological cells are attached to biomolecules initially adsorbed on the surface; composition and configuration of these adsorbed biomolecules conduct signals to cells determining their behavior. Thus, engineering the surface in nanometer scale has been inferred as an attractive technique to manipulate surface characteristics in order to control the absorbance of biomolecules in this scale and improving the cell behavior $[4,5]$.

Traditional methods for altering the surface in nanoscale require multi-step processes, they are chemically complicated or result in uncontrolled surface property changes[6]. A new technique, severe plastic deformation (SPD), is an alternative approach that can produce metals with ultra-fine grain sizes through applying large deformation at high strain rates. SPD techniques do not have complications of other nanofabrication methods and also increase the mechanical strength of the bulk material[7]. Linear plane-strain machining (LSM) is a SPD method whereby a sharp tool quickly removes the surface metal in a predetermined depth and angle. In this method, average grain size can be controlled by three parameters of machining tool angle, tool velocity, and cutting depth[8].

In this study, we studied the MC3T3 (an osteoblast precursor cell line) cellular responses on the austenitic 316L stainless steel samples with three different nanoscale average grain sizes obtained by linear plane-strain machining SPD technique. In addition, two types of oxide layers were grown on various nanograin surfaces (i.e., chemical and thermal treatment) to investigate the role of the nanocrystal structures both on surface oxide formation and surface biological function, as well as to compare the MCT3T3 cell adhesion. 


\section{Materials and Methods}

Commercially available, annealed and cold-finished 316L stainless steel plate (McMASTER-CARR, Cleveland, OH, USA) was processed by linear plane-strain machining at room temperature using cutting depth of $150 \mu \mathrm{m}$ and tool velocity of $25 \mathrm{~mm} / \mathrm{s}$ for three different rake angles of $+40^{\circ},+20^{\circ}$ and $0^{\circ}$. Then, the samples were mechanically and chemically finished to a mirror-finish surface. Oxide layer was chemically grown on all three nanograin samples and the control sample (i.e., coarse-grain samples) using the chemical method reported by Evance et al.[9]. Thermal treatment was also examined to grow the oxide layer on nanograin samples and the control sample via oxidizing the samples in air at $1000^{\circ} \mathrm{C}$ temperature for $15 \mathrm{~min}$ and rapidly cooling down to the room temperature[10]. Table 1 shows the list of samples prepared in this study using LSM technique along with the surface treatment.

\begin{tabular}{|c|c|c|c|c|c|c|c|c|c|c|c|c|}
\hline Sample \# & 1 & 2 & 3 & 4 & 5 & 6 & 7 & 8 & 9 & 10 & 11 & 12 \\
\hline Rake angle $\left(^{\circ}\right)$ & 0 & 0 & 0 & 20 & 20 & 20 & 40 & 40 & 40 & $\mathrm{~N}$ & $\mathrm{~N}$ & $\mathrm{~N}$ \\
\hline Surface treatment & $\mathrm{N}$ & Chem & Therm & $\mathrm{N}$ & Chem & Therm & $\mathrm{N}$ & Chem & Therm & $\mathrm{N}$ & Chem & Therm \\
\hline
\end{tabular}

Surface topography and microstructure of all samples was characterized by scanning electron microscopy (SEM) (Jeol-JSM 6610 LV, Japan). Surface chemistry of all samples before and after thermal and chemical treatment was analyzed using X-ray photoelectron Spectroscopy (XPS, ESCALAB 250 XI Thermoscientific, USA). MC3T3 cells at passage number 6 were seeded on the specimens at a density of $3 \times 10^{5}$ cells/ml. Fluorescent images were taken to observe the live and attached cells using fluorescent microscopy (Olympus BX43, Japan) using Calcein AM (life technologies ${ }^{\mathrm{TM}}$, NY, USA) staining. Besides, the viability and proliferation of cultured cells was determined using MTT (3-[4,5-dimethylthiozol-2-yl]-2,5diphenyltetrazolium bromide) (Millipore, MA, USA) colorimetric assay in different time periods ( $1 \mathrm{hr}, 2 \mathrm{hrs,}$ 4hrs, 1 day and 5 days).

\section{Results}

\subsection{Surface Characterization}

Figure 1 shows the results of SEM imaging on all the samples listed in table 1 . Surfaces with native oxide layer (i.e., no grown oxide either chemically or thermally) showed smooth and mirror-finish topography in micrometer range and no noticeable difference was observed in the surface topography of control sample and the samples obtained by different rake angles (Figure 1A1, 1B1, 1C1 and 1D1). However, island-like 
structures, grooves and holes are appeared on the surface after $1 \mathrm{hr}$ chemical treatment. The average size of these island-like structures was reduced and the surface roughness increased over decreasing the rake angle (Figure 1A2, 1B2, 1C2 and 1D2). After thermal treatment, new micro-features were appeared on the surface; however, the size and shape of them were substantially smaller than those observed in the chemically treated samples (Figure 1A3, 1B3, 1C3 and 1D3).

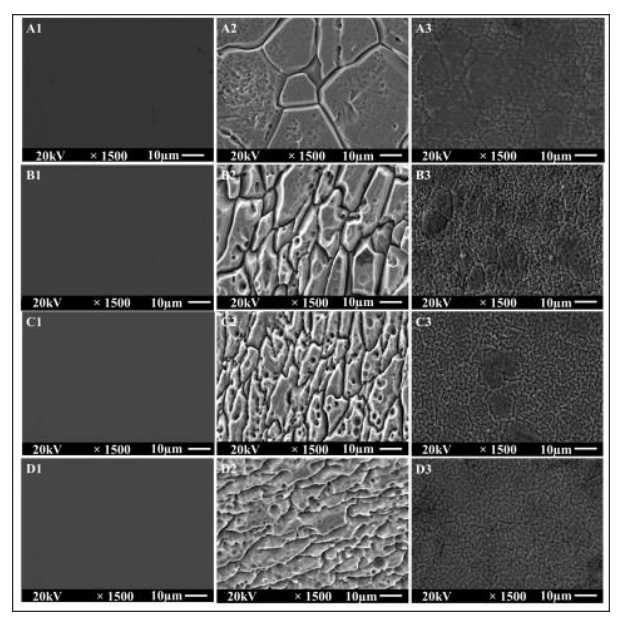

In addition to surface topography, the chemical compositions of samples were analyzed using XPS technique. Figure $2 \mathrm{~A}, 2 \mathrm{D}$ and $2 \mathrm{G}$ show the survey spectra of all samples before and after chemically and thermally surface treatment, respectively. Survey spectra did not change over changing the rake angle before and after chemical and thermal surface treatments. The survey spectra of untreated surfaces showed dominating peaks corresponding to elements of carbon, oxygen, chromium and iron. The lower peak corresponded to the nickel. High resolution spectra of $\mathrm{Fe} 2 \mathrm{p}$ and $\mathrm{Cr} 2 \mathrm{p}$ in untreated surfaces are presented in Figure $2 \mathrm{~B}$ and Figure 2C, respectively. $\mathrm{Fe} 2 \mathrm{p}$ spectra represented a double peak corresponding to free iron and iron oxide compositions. The XPS results for Cr2p region also indicated the presence of free chromium and chromium oxide compositions on the surface. In both chemically and thermally treated samples, peaks corresponding to free iron and chromium disappeared (Figure 2E, 2F, 2H and 2I). In chemically treated surfaces, similar peaks to the peaks in the untreated surface were observed; however, the peaks corresponding to chromium oxide and iron oxide were significantly intensified. In thermally treated surfaces, in addition to present peaks observed in untreated surfaces, a new peak corresponding to manganese compositions appeared on the surface and the peak corresponding to chromic oxide was strongly intensified. 


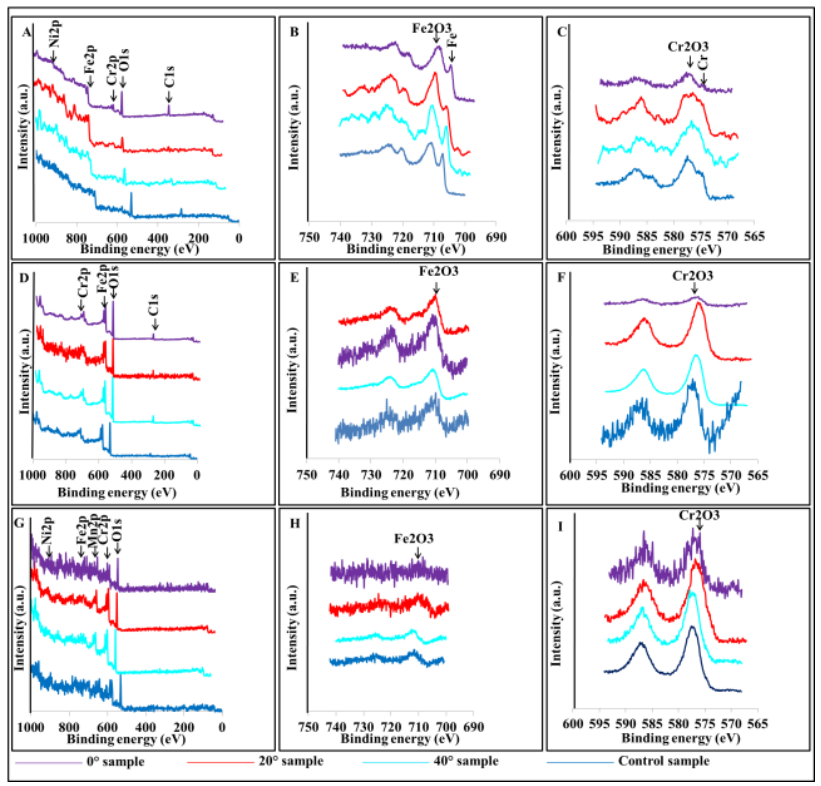

\subsection{Cell Culture Study}

The results of MTT assay for viability of MC3T3 cells on different substrates over different time periods ( $1 \mathrm{hr}$, 2hrs, 4hrs, 24hrs and 5 days) are shown in Figure 3. Figure 4 shows Calcein AM stained live cells cultured on all the samples before and after surface treatments. Over decreasing the rake angle in untreated samples, MTT results show that cell viability increases in all the time periods (Figure 3A) and the fluorescent intensity also became higher (Figure 4A1, 4A2, 4A3 and 4A4); the highest number of live cells was spread out on the untreated $0^{\circ}$ surface (Figure 4A4). In the chemically treated surfaces, no significant difference is observed between the viability of cells in different time periods (Figure 3B), besides, the fluorescent imaging also shows no significant difference in the number of live cells in chemically treated surfaces (Figure 4B1, 4B2, 4B3 and 4B4). In thermally treated surfaces, both MTT assay and fluorescent imaging results show that the number of live cells is higher in $0^{\circ}$ sample compared to the control sample (Figure $3 \mathrm{C}$ and Figure $4 \mathrm{C} 1,4 \mathrm{C} 2$, 4C3 and 4C4). The cell growth on thermally and chemically treated surfaces is noticeably smaller compared to the analogous untreated surfaces.

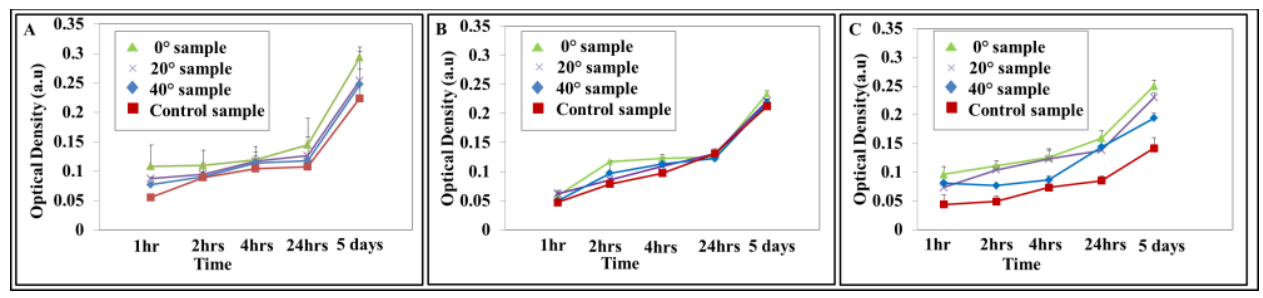




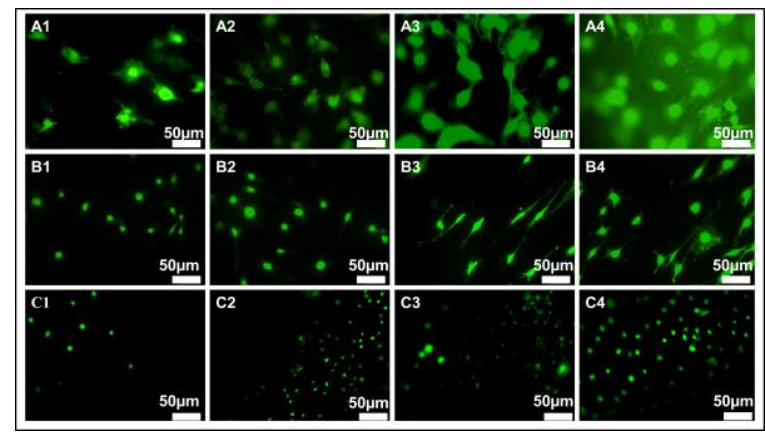

\section{Discussion}

Linear plane-strain machining is a two-dimensional SPD technique which can impose a high strain rate altering the material microstructure under a precisely controlled process via varying parameters of rake angle $(\alpha)$, depth of cut and tool velocity. The average grain size of 316L stainless steel is about $22 \mu \mathrm{m}$ while after plane-strain machining, it reduces to nanometer scale (e.g., $42 \mathrm{~nm}$ in $0^{\circ}$ rake angle)[11].

XPS spectra revealed no change in the chemical composition of the surface after SPD process of the planestrain machining implying that refining the average grain size down to nanoscale range did not change the chemical composition of grown oxides. In XPS spectra of untreated surfaces, the presence of peaks corresponding to $\mathrm{Fe}$ and $\mathrm{Fe}_{2} \mathrm{O}_{3}, \mathrm{Cr}_{2} \mathrm{O}_{3}$ and nickel oxide compositions showed that an oxide layer formed on the surface[12]. After both chemical and thermal treatment, the peaks corresponding to the elemental Fe and Cr disappeared implying growing the oxide layer on the surface. In our study, XPS results of thermally treated samples showed in addition to the presence of peaks corresponding to $\mathrm{Fe}_{2} \mathrm{O}_{3}$ and $\mathrm{Cr}_{2} \mathrm{O}_{3}$, a new peak appeared in $638-648 \mathrm{eV}$ region corresponding to manganese enrichment on the surface[10]. It is expected that growing a stable surface oxide enhances the corrosion resistance and prevents toxic ion release resulting in improving the biocompatibility but in thermally and chemically treated surfaces, cell viability decreased compared to the native stainless steel oxide. The oxide film formed by chemical treatment results in an island-like structure with a non-uniform chemical composition and porosity within its depth (chromium-enriched surface). Therefore, chemically formed oxide on stainless steel reduces the corrosion resistance compared to the passive oxide naturally formed on the surface[13]. In thermally treated surface, reduction in cell viability may refer to changing the surface topography as well as the appearance of manganese oxide composition on the surface[14]. Wang et al., demonstrated that nanograin 316L stainless steel treated under $1000^{\circ} \mathrm{C}$ annealing 
temperature for longer time than we used (i.e., 8hrs) was noticeably stable and the grain size slightly changed[15]; however, more studies is also required to elucidate the effect of annealing process on the microstructure in our case. Our future studies are directed to study the microstructure of the oxide layers using transmission electron microscopy (TEM) analysis and evaluate the in vivo response of nanograin substrates as well.

\section{Conclusion}

The aim of this paper is to study the interaction of MC3T3 cells with the oxide layers formed on nanograin substrates naturally, chemically and thermally. Linear plane-strain machining presents a suitable SPD technique to develop samples with ultrafine average grain size and controlled microstructure. Refining the average grain size coupled with surface treatments created surfaces with a wide range of surface characteristics variations including different surface topography and chemistry. Grain size refinement did not reveal any difference in chemical composition of the native oxide as well as thermally and chemically grown oxides on nanograin samples compared to coarse-grain samples. After chemical treatment, a chromiumenriched oxide was formed on the surface while after thermal treatment, the surface oxide with increasing amount of manganese and chromium compositions appeared on the surface. The primary in vitro results suggested untreated $0^{\circ}$ sample as a promising surface for improving osteoblast cell interaction.

\section{Acknowledgment}

The authors most gratefully appreciate funding provided for this work by NSF under award number 1233909 and University of Pittsburgh Central Research Development Fund.

\section{References}

[1] Kurtz S, Ong K, Lau E, Mowat F, Halpern M. Projections of primary and revision hip and knee arthroplasty in the United States from 2005 to 2030. The Journal of Bone \& Joint Surgery. 2007;89:780-5. [2] Navarro M, Michiardi A, Castano O, Planell J. Biomaterials in orthopaedics. Journal of the Royal Society Interface. 2008;5:1137-58.

[3] Niinomi M. Metals for biomedical devices: Elsevier; 2010.

[4] Boyan BD, Hummert TW, Dean DD, Schwartz Z. Role of material surfaces in regulating bone and cartilage cell response. Biomaterials. 1996;17:137-46.

[5] Shayan M, Jung Y, Huang P-S, Moradi M, Plakseychuk AY, Lee J-K, et al. Improved osteoblast response to UV-irradiated PMMA/TiO2 nanocomposites with controllable wettability. Journal of Materials Science: Materials in Medicine. 2014;25:2721-30.

[6] Sun L, Berndt CC, Gross KA, Kucuk A. Material fundamentals and clinical performance of plasma-sprayed hydroxyapatite coatings: a review. Journal of biomedical materials research. 2001;58:570-92. 
[7] Valiev RZ, Estrin Y, Horita Z, Langdon TG, Zechetbauer MJ, Zhu YT. Producing bulk ultrafine-grained materials by severe plastic deformation. Jom. 2006;58:33-9.

[8] Idell Y, Facco G, Kulovits A, Shankar M, Wiezorek J. Strengthening of austenitic stainless steel by formation of nanocrystalline $\gamma$-phase through severe plastic deformation during two-dimensional linear planestrain machining. Scripta Materialia. 2013;68:667-70.

[9] Evans T, Hart A, James H, Smith V. New process for colouring stainless steel. Trans Inst Metal Finishing. 1972;50:77-9.

[10] Guillamet R, Lopitaux J, Hannoyer B, Lenglet M. OXIDATION OF STAINLESS-STEELS (AISI-304 AND AISI-316) AT HIGH-TEMPERATURE-INFLUENCE ON THE METALLIC SUBSTRATUM. JOURNAL DE PHYSIQUE IV. 1993;3:349-56.

[11] Shekhar S, Cai J, Basu S, Abolghasem S, Shankar MR. Effect of strain rate in severe plastic deformation on microstructure refinement and stored energies. Journal of Materials Research. 2011;26:395-406.

[12] Hryniewicz T, Rokosz K, Rokicki R. Electrochemical and XPS studies of AISI 316L stainless steel after electropolishing in a magnetic field. Corrosion Science. 2008;50:2676-81.

[13] Stoychev D, Stefanov P, Nicolova D, Valov I, Marinova T. Chemical composition and corrosion resistance of passive chromate films formed on stainless steels $316 \mathrm{~L}$ and 1.4301 . Materials chemistry and physics. 2002;73:252-8.

[14] Park J-W, Kim Y-J, Jang J-H. Surface characteristics and in vitro biocompatibility of a manganesecontaining titanium oxide surface. Applied Surface Science. 2011;258:977-85.

[15] Lee YB, Shin DH, Park K-T, Nam WJ. Effect of annealing temperature on microstructures and mechanical properties of a $5083 \mathrm{Al}$ alloy deformed at cryogenic temperature. Scripta Materialia. 2004;51:3559 .

\section{Figures captions}

Figure 1. SEM images of A) Control sample: 1. Untreated, 2. Chemical treated, 3. Thermal treated, B) $0^{\circ}$ sample: 1. Untreated, 2. Chemical treated, 3. Thermal treated, C) $20^{\circ}$ sample: 1. Untreated, 2. Chemical treated, 3. Thermal treated and D) $40^{\circ}$ sample: 1 . Untreated, 2. Chemical treated, 3. Thermal treated

Figure 2. Survey XPS spectra (A) and high resolution XPS spectra of Fe $2 p(B)$ and $\mathrm{Cr} 2 p(C)$ for control sample and $0^{\circ}$, $20^{\circ}$ and $40^{\circ}$ samples, Survey XPS spectra (D) and high resolution XPS spectra of Fe2p (E) and Cr2p (F) for control sample and $0^{\circ}, 20^{\circ}$ and $40^{\circ}$ samples after chemical treatment, Survey XPS spectra (G) and high resolution XPS spectra of $\mathrm{Fe} 2 \mathrm{p}(\mathrm{H})$ and $\mathrm{Cr} 2 \mathrm{p}(\mathrm{I})$ for control sample and $0^{\circ}, 20^{\circ}$ and $40^{\circ}$ samples after thermal treatment.

Figure 3. Viability of MC3T3 cells incubated in time periods of $1 \mathrm{hr}, 2 \mathrm{hrs}$, $4 \mathrm{hrs}, 24 \mathrm{hrs}$ and 5 days in $0^{\circ}, 20^{\circ}, 40^{\circ}$ and control untreated samples (A), chemically treated $0^{\circ}, 20^{\circ}, 40^{\circ}$ and control samples (B), thermally treated $0^{\circ}, 20^{\circ}, 40^{\circ}$ and control samples (C) tested by MTT assay (data presented as mean $\pm \mathrm{SD}, \mathrm{n}=3$ and $\mathrm{P}$-value $<0.01$ )

Figure 4. Fluorescent images of Calcein AM stained MC3T3 cells after 48hrs culturing on A1) Control surface, A2) $40^{\circ}$ sample, A3) $20^{\circ}$ sample, A4) $0^{\circ}$ sample, B1) Chemically treated control Surface B2) Chemically treated $40^{\circ}$ sample, B3) Chemically treated $20^{\circ}$ sample and B4) Chemically treated $0^{\circ}$ sample, C1) Thermally treated control surface, C2)

Thermally treated $40^{\circ}$ sample, C3) Thermally treated $20^{\circ}$ sample and C4) Thermally treated $0^{\circ}$ sample

\section{Table caption}

Table 1. List of samples prepared by linear plane-strain machining (LSM) technique with different rake angles (i.e., $0^{\circ}, 20^{\circ}$ and $40^{\circ}$ ) and after surface treatment (i.e., chemical treatment, thermal treatment) (Chem: chemical treatment, Therm: thermal treatment and $\mathrm{N}$ : none) 